\title{
Collective Housing and Residential Quality-Four Complexes of Low, Medium and High Density in Córdoba, Argentina
}

\author{
M. Cecilia Marengo ${ }^{1,2}$ \\ ${ }^{1}$ National Council for Scientific and Technological Research, Argentina \\ ${ }^{2}$ National University of Córdoba, Argentina \\ Email: mcmarengo@gmail.com
}

How to cite this paper: Marengo, M.C. (2019) Collective Housing and Residential Quality-Four Complexes of Low, Medium and High Density in Córdoba, Argentina. Open Access Library Journal, 6: e5477. https://doi.org/10.4236/oalib.1105477

Received: May 17, 2019

Accepted: June 14, 2019

Published: June 17, 2019

Copyright $\odot 2019$ by author(s) and Open Access Library Inc.

This work is licensed under the Creative Commons Attribution International License (CC BY 4.0).

http://creativecommons.org/licenses/by/4.0/ (c) (i) Open Access

\begin{abstract}
The study addresses an evaluation of the habitability conditions in collective housing from the perspective of building density and residential quality. The methodological proposal considered a multivariate approach. Initially, a simulation model was formulated integrating different situations in a residential quality index (density, scale, number of inhabitants). In this phase, a qualitative approach was incorporated to account for the perception of the inhabitants. The research question is: How density values are translated into satisfaction/dissatisfaction with a residential model? Four housing complexes of low, medium and high density, of public production in Córdoba city, Argentina are reinterpreted in light of the qualitative analysis, based on the development of interviews with key informants and observationson-site. The conclusion of the study allows new openings in the discussion of density values and residential quality, useful when planning housing interventions.
\end{abstract}

\section{Subject Areas}

Sociology

\section{Keywords}

Density, Residential Quality, Collective Housing, Residential Satisfaction

\section{Introduction}

In the research carried out in the period 2016-2017 [1], we proposed to study models of collective housing and provide a comparative evaluation of habitability conditions, from the perspective of building density and residential quality. 
The study focused on residential densification models that appear in the consolidated urban tissues. The cases were integrated with 15 collective housing complexes, which differ in terms of building density and urban form, located in the city of Córdoba, Argentina.

The objective was to investigate which levels of density present the housing complexes and which are more suitable from the perspective of the residential quality implicit in each project.

The density values of the complexes present a compact land use in comparison to suburban residential developments that materialized in the last decades. We ask ourselves: to what extent a denser occupation form can offer quality and be an alternative to the dominant model of urban expansion that materializes socially homogeneous peripheries, physically fragmented and diffuse in terms of urban-land use? The methodological proposal considered a multivariate approach. A preliminary simulation model was formulated as a tool, which allows integrating different situations of density, scale, number of inhabitants and other indicators, which account for the complexity in the concept of residential quality, linked to density.

In this phase of the research, we make more complex the model of quantitative perspective initially formulated. To do this, we incorporate a qualitative approach that seeks to account for the perception of the inhabitants in the assessment of the residential model. We ask ourselves: How are the values of building density translated into the satisfaction/dissatisfaction that is attributed to a certain form of physical-spatial organization? What dimensions are significant from a qualitative assessment in the housing proposals?

To answer these questions, four housing complexes of low, medium and high density of public production located in the city of Córdoba, Argentina are evaluated in the analysis. The quantitative analytical perspective is reinterpreted in the light of qualitative analysis, based on the development of interviews with key informants and on-site observation. Finally, the conclusion of the study allows new openings in the subject of density and residential quality, useful when planning new housing interventions.

\section{Urban Growth and Approaches to Density}

The residential developments that appear in the cities in the last decades show the predominance of an extended and dispersed urban growth pattern, where at the same time the consumption of urbanized land per inhabitant increases, the gross density in urban areas decreases. This condition, present both in developed and developing countries, [2] has been the focus of research in different urban studies and has led to question this form of growth pattern as unsustainable. From a perspective focused on the physical planning of growth, density is proposed as a viable indicator to account for the effectiveness of different models of land use and is linked to the production of collective housing.

Density is defined as the number of inhabitants or dwelling units per area 
(hectares, in the case of urban scale studies, or square kilometers in the case of territorial planning scale). Although this measure indicates the intensity of land use of an area, (housing per hectare, inhabitants per hectare) the issue of density in the field of urban planning responds to different visions and approaches to sustainability. In some cases the focus is placed on the building morphology (urban form), for example in the case of comparative studies between different models of residential housing (towers, blocks, or mixed forms) with the same density and differing in the building typology.

Other studies emphasize the concept of minimum occupation thresholds necessary for urban functions to be viable and analyze the vitality of the fabric, the mix and intensity of uses, among other aspects. Other approaches advocate the need to achieve greater efficiency in land use and occupation. In this case, density values are linked to a more efficient occupation of urban land and promote more compact building forms (understanding that urban land is a scarce resource). The advantages consider how it would contribute in terms of economy in the provision of support infrastructures and the viability and efficient distribution of urban facilities (transport networks and public services). Finally, other perspectives link density with the sustainability of growth, as is the case of studies of urban mobility, energy efficiency and environmental issues associated with the emission of pollutants.

The perspective we develop in the research links Density with the concept of Residential Quality. We understand that to decide if a density value is adequate, we must consider quantitative and qualitative parameters that relate the physical-spatial forms of a habitat with the demands, preferences and expectations of a social group, which are evidenced in a particular way of using residential space. From this perspective, density is not only a descriptive or indicative value of the relationship: inhabitants per hectare or dwellings per hectare, but it also has an operational dimension, whose importance lies in the ability to provide a prospective orientation at the time of physical planning of human settlements.

\section{Density and Residential Model}

Density has been a recurring theme in urban research since the postwar reconstruction period in the 20th century. It has been approached from the perspective of the planning indicators that allowed reaching more efficient forms in land use occupation for a growing residential demand, which had to be addressed through public housing policies. At the beginning of the past century in Europe, research began to study the types of building, and forms of aggregation of housing units and different alternatives in collective housing were developed. Different settlement models were recognized, based on the use of specific typologies in low, medium and high density, and their combinations in height (high height and density, medium height and high density, etc.). As mentioned by [3] "the model of the Hoff comes with the superblock of housing self-sufficient lying inserted in the frame of the existing city and was opposed to the Siedlung of low 
building density that colonized the outer territory in the urban perimeter".

Research on building types and density were also views on the forms of urban growth that should be promoted in the new urbanized extensions in the central countries, in a context characterized by the housing deficit. To the dominant models of low density individual housing in the peripheries (garden city) and collective housing in high density towers in central areas (dense city), it was added the model of half height, medium density, which becomes a topic in the seventies and eighties as one of the most efficient combinations to meet an urban response to the housing deficit, with average densities ranging from 60 to 80 dwellings per hectares [4].

The debate on the city and urban growth, (dispersed city - compact city) is partly focused on the issue of density and forms of occupation. The density represents an inverse index to the consumption of urban land, is expressed in the ratio of inhabitant/hectare and supposes a more rational use of the resources, both natural and urban, which for the state means a greater economy in the provision of services and urban infrastructures. On the other hand, for the private sector, density is translated and operated as a volume of transactions that provides greater profitability and profit.

A categorization of density, such as that presented by [3] identifies at least 5 ranges to catalogue collective housing projects in developed countries, linked to a specific urban location and associated with more or less compact forms of land use.

Values in a range between 50 dwellings/hectares to more than 300 dwellings/ hectares are associated with:

- Low-density suburban developments and individual housing, less than 50 dwellings/hectares,

- Peripheral urban developments in low density, between 50 - 100 dwellings/hectares,

- Developments in medium density, in consolidated or historical areas, between 100 - 200 dwellings/hectares,

- Developments in high density in central areas or new suburban centralities, between 200 - 300 dwellings/hectares,

- New interventions of housing towers with marked vertical development and very high density, more than 300 dwellings/hectares.

In Latin American cities and Córdoba in particular, there are significant variations in the densities of the urban form, which derive from the growth process itself and the socio-economic development conditions that have characterized the production of residential space.

- The case of individual housing less than 50 dwellings/hectares, include both developments in the real estate market in suburban extensions for very high income sectors (closed urbanizations, and special residential developments) and public housing projects intended for very low income sectors (in extensions with peripheral or edge locations). In the developments of private neighborhoods of high standard, net density ranges from 6 - 10 dwel- 
ling/hectares, (in plots whose minimum size is $1000 \mathrm{~m}^{2}$ ), or $18-20$ dwelling/hectares, closed neighborhoods with plots whose minimum size is 600 $\mathrm{m}^{2}$. In the second case, public developments called neighborhoods-cities (by the scale and levels of equipment they present) respond to mass relocation programs of poor population, and have a net density of $40 \mathrm{dwelling} / \mathrm{hectares}$, with plots whose minimum size is $250 \mathrm{~m}^{2}$. In individual housing plans developed by cooperatives, (open and mono-functional neighborhoods) the net density ranges from 40 to 50 dwelling/hectares and plots varies from 200 to $250 \mathrm{~m}^{2}[5]$.

- In the case of collective housing, we focus the object of study of the research to collective housing complexes. The last is understood as a group of dwellings conceived within an integral concept, generally approved as a single project or program by the pertinent public authority, almost always within the shared horizontal property format [6]. It is a unit planned by the State (or by private agents) to respond to the housing problem. It is characterized as a morphological and organizational unit with a recognizable structure, common spaces and facilities, with identity and sense of belonging by its inhabitants [7].

The research deals with the study of housing complexes materialized in the city of Córdoba over a long period of time, with interventions of public production (in the period 1970-1990) and private(in the period 1990-2010). The significant fact of this type of housing complexes is that they are isolated operations. The significant presence in the urban context comes fundamentally from the formal model. The density of the projects is in clear contrast with the urban fabric in which they are located. This particular condition allows us to analyze them as singular cases, with varying levels of densification in a range between 58 dwelling/hectares, to 499 dwelling/hectares.

For some authors [8] density is the most relevant variable, but not the only one to improve urban quality, recognizing the importance of the existence of other uses related to housing, such as green public spaces or urban equipment. These studies propose minimum density values that "assure" the intensity of uses and relationships, while at the same time they allow generating a critical mass that makes public transport and service networks more efficient. Although it is usual to approach density studies from quantitative perspectives, these have their correlate in the forms of uses, appropriation and perception by the inhabitants. In the last period (21st century) it is observed that density has become the most relevant attribute to define the characteristics of a new type of private management interventions, where the obsession to reach the greatest number of dwellings is what is defining the urban form, with a new paradigm that promotes urban densification but in an isolated and closed way with respect to its immediate surroundings.

\section{Quality of Life and Residential Satisfaction}

The concept of quality of life is associated with the assessment and personal 
perception that each individual has about their habitat and is usually linked to another concept: residential satisfaction. The theoretical background can be traced in the sociological studies on housing, developed by the Chicago School, which imprinted an approach that overcomes the hygienist vision developed in the 19th century. In the twentieth century, economists' criteria guided the construction of public housing, without considering the residential satisfaction of the inhabitants, as an indicator that could provide feedback to the project process.

As [9] mention at this stage, social and spatial preferences about housing are determined by social, political, economic and historical forces and rarely consider the valuations of individuals. The approaches on the quality of life as an analytical category applicable to the study of residential interventions began to spread in Europe from the seventies as a way to counteract the exclusively economistic analysis, also questioning the model of economic development and the consumer society. In previous studies we define residential quality as the set of attributes available to a settlement to meet the needs (objective and subjective) of a population. It is mediation between aspirations and lacks (objective deficiencies), available resources and material conditions to meet them. The Habitat I Conference in Vancouver (1976) defines housing not only as a physical unit that houses a family (its characteristics of space and internal equipment) but as the conjugation of the inside and the outside, complex of the social and individual, of the public and the private. The conception extends to the land, infrastructure, urbanization and the services available to it, the social and community equipment, in a specific cultural, socio-economic, political and environmental physical context. Social needs have an anthropological foundation, security, openness, certainty, adventure, isolation, encounter; human needs that led to the collective are presented as the need of the city [10]. A residential area not only covers the interior of the home, but also the physical and social space where it is inserted. Although residential quality can be objectified through the characterization and definition of physical and social attributes, these become subjective when assessed by residents (residential satisfaction/dissatisfaction).

From this conception of the habitat, residential satisfaction involves at least three scales of analysis: the housing unit (physical characteristics of housing, requirements and aspirations of households); the housing complex (in this case, the dialectic between the private space and the collective space of the complex acquires particular importance) and the neighborhood in which it is located (understood as a scale of urban-sectorial relationship).

When the object of study is collective housing, the dynamics of social and neighborhood life are constituted in a different way that the case of individual housing. There is a need to contemplate the environment where the building is located. The social relations that are built with the neighbors in common spaces for collective use in their interior including shops and other equipment, becomes important. The interactions, encounters, belongings, appropriations, are marked by other logics, giving a certain degree of autonomy to each housing complex 
and influencing the assessments, perception and satisfaction that each resident makes of its own environment.

Taking into account this last aspect, residential quality as mentioned in [11] is considered as: "The perception and assessment that various observers and participants assign to the component factors of a human settlement, with their mutual interactions and with the context where it is inserted, establishing different hierarchies according to variables essentially of social, cultural, economic and political nature".

In the assessment of residential quality come into consideration the objective conditions of space, such as physical environments, the structures of the housing complexes, the typology of dwellings, services and infrastructure, among other quantitative aspects and the subjective conditions or perception that residents have. Residential satisfaction can be understood as the attitude or affect produced by living in a certain context. As mentioned in [12] is the "level of liking or dislike that people feel for the environment where they live, including housing and their environment". This subject-space interaction results in positive or negative feelings that must be valued and considered when designing housing interventions.

\section{Methodological Approaches for a Qualitative Evaluation}

To inquire about collective housing projects and the relationship between Density-Residential Quality, we addressed the study of 15 proposals and developed a comparative analysis. Applying the simulation model tool, we obtained a Residential Quality Index for each housing complex, based on quantitative indicators.

To incorporate the qualitative analytic approach and to make the evaluation more complex, we developed various approach techniques such as interviews with key informants, observation in the sites and a survey. The cases of study were five housing complexes, of which we present four in this article, all of them built by of public investments. The objective in this phase of the research is to reconstruct the way in which residents perceive the analytical dimensions previously defined in the quantitative approach.

We inquired about the family composition, housing tenure, and an approximation to the socio-economic level of the interviewees. Then, questions of a qualitative nature were formulated, including aspects on: the housing unit (size, typology, expectations in relation to the existing areas); the housing complex (planned parking spaces, spaces for collective use, levels of sociability among the inhabitants) and the neighborhood and its surroundings (urban location and accessibility to nearby facilities, safety conditions, among others).

\section{Four Study Cases of Housing Complexes: "Hogar Propio", "SEP", "Cispren" and "IPV Juniors"}

Figure 1 summarizes the values obtained for the qualitative indicators surveyed 
in the four complexes, which represent low, medium and high density urban forms. It is worth mentioning that although they are all of public production, they were built in different decades, with orientations and dissimilar dominant models in the production of social housing in terms of design and planning forms. There are parallel blocks (Hogar Propio Complex), succession of semi-closed city blocks (Complex SEP II), closed city blocks (that occupy the perimeter of the block-CISPREN Complex) and combination of towers and blocks (IPV Juniors Complex). The complexes differ not only in densities but in their size, measured by the number of dwelling units. They are integrated with 841 dwelling units in the case of SEP (the largest scale and lowest density); with 444 dwelling units IPV Juniors (which is the highest density), with 150 dwelling units in the case of Cispren and 112 units in Hogar Propio Complex, the smallest.

In terms of urban location, they are placed in the pericentral and peripheral areas, with a displacement time to the center (measured in public transport) that varies between 36 to 51 minutes respectively. The peripheral areas that at the time of its materialization were in the city borders today are totally integrated to the urban structure due to the process of urban growth.

The results of the comparative evaluation of the proposals, in terms of the quantitative dimensions considered in the study, are synthesized in the value of the Residential Quality Index (RQI). This varies between 0.47 in the case of higher quality (Hogar Propio Complex) and 0.26 in the case of lower quality

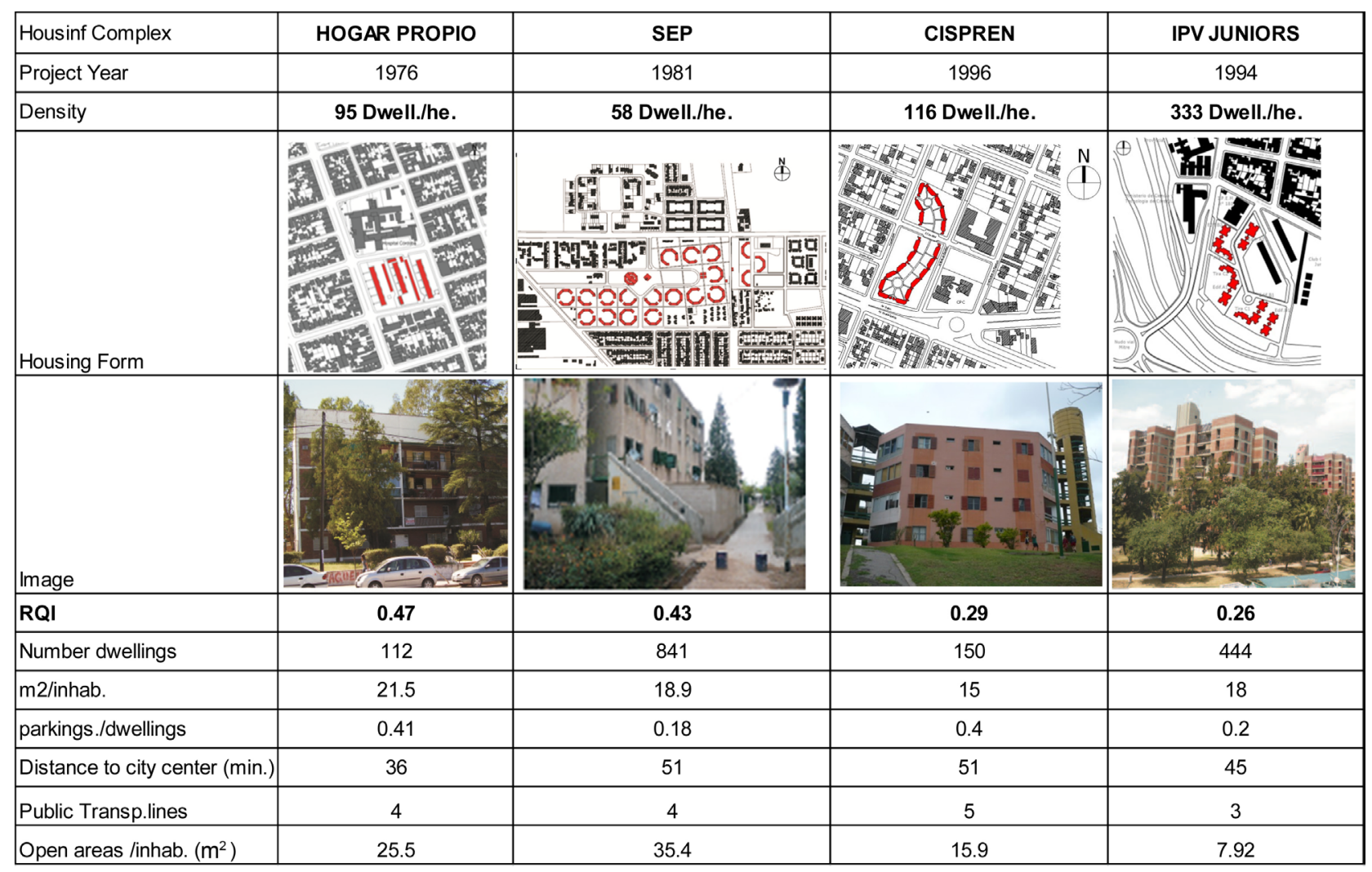

Figure 1. Synthesis of quantitative indicators in four housing complexes. Own elaboration. 
(IPV Juniors Complex).

The index built and integrated into a simulation model contains the following quantitative dimensions:

- The square meters of residential area per inhabitant (that is the size of the housing unit, but not as an absolute figure, but calculated according to the number of inhabitants by type). This value assumes ranges between 15 and $21,5 \mathrm{~m}^{2}$ per inhabitant, with a direct impact on the RQI considering that the residential satisfaction is greater when there is more living space per inhabitant in the dwelling unit.

- The index of parking spaces by number of dwellings. In general, the values obtained are low, given that the public production of housing did not foresee parking spaces in the original design, and when it did it was much lower than the current demand that was revealed in the housing complexes.

- The mobility measured in minutes on public transport to the city center, and the accessibility depending on the amount of public transport lines in the area of the housing complexes. The slight differences registered in the quantitative analysis are then interpreted according to the composition of the households, life family cycle and socio-economic segmentation of the inhabitants, as well as the mobility preferences of the inhabitants in public transport or in private car.

- The area of collective open space per inhabitant that presents each proposal differs significantly in a range that goes from $8 \mathrm{~m}^{2}$ (IPV Juniors Complex) to $35 \mathrm{~m}^{2}$ (SEP Complex). Although this is a significant indicator in collective housing assemblies, when we want to establish a value of adequacy or a dimensional reference, subjective variables must be considered (such as identity, appropriation, use patterns, security, among others). In these take part the degree of delimitation of the spaces, the different scales of use, the social context and the institutional organization for the maintenance of the same.

\section{Results Obtained}

\subsection{Hogar Propio Complex}

As Figure 2 shows, Hogar Propio Complex has a middle value of density and the highest value of the RQI. Integrated by Families (80\%); of 4 or more members (60\%); mostly tenants (67\%), medium socio-economic level (53\%) and medium high socio-economic level (20\%), Figure 2.

The size of the house is considered adequate to the family group in $92 \%$ of cases, they are large apartments occupied by numerous family groups, presenting a correlation between the quantitative assessment of the indicator of residential area by inhabitants (which reaches the highest value in this complex) and the qualitative assessment in terms of satisfaction expressed by the residents.

The same is observed when analyzing the parking, it is considered adequate in 93\% of cases (although the project do not considers a high number of parking spaces). The parking area was extended by the residents, occupying the open 


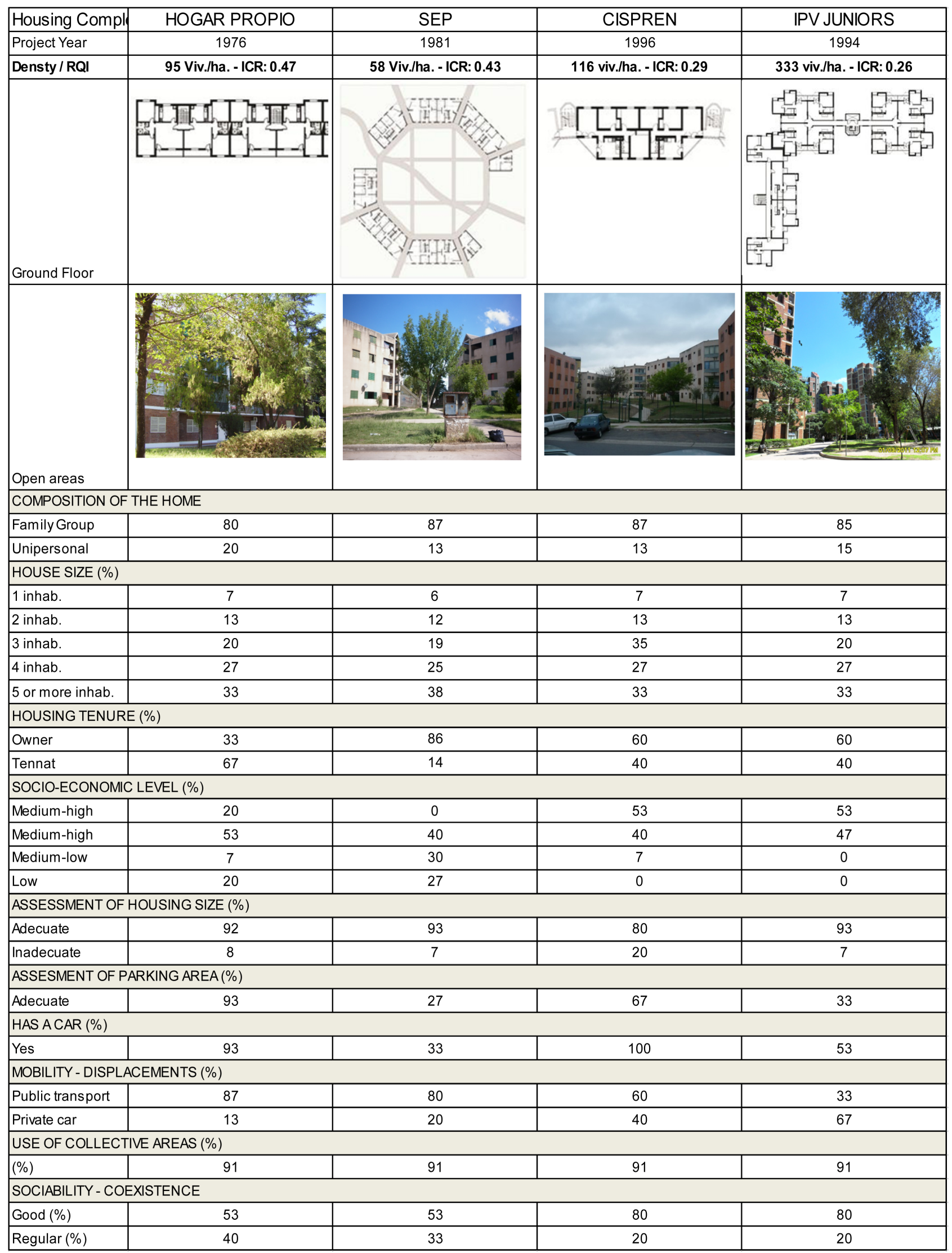

Figure 2. Summary of qualitative assessment in four housing complexes. Own elaboration. 
common spaces. Although the value of those with have a car is high (93\%), there is a high value of accessibility and mobility through the use of public transport (87\%). This aspect is highly well-valued by residents, given the situation of location of the complex on a main avenue (corridor of the urban structure). It is very connected with different neighborhoods and has different public transport lines nearby. Regarding the assessment of the open areas in the complex, $91 \%$ mentioned that uses it both, the garden towards the perimeter streets and the internal space delimited by the parallel blocks. This area was closed and equipped by the residents (seats and vegetation, among others), observing high levels of sociability and appropriation (53\%). It is a housing complex built in the 70 s, which is maintained in good building conditions and is required to rent by family groups given the advantages offered by the type of departments (large, comfortable) the urban location and the conditions of accessibility.

\subsection{SEP II Complex}

In the case of SEP II Complex, it has a low density value and a middle value of RQI.

It is the largest residential complex group, composed of families (87\%); of 4 or more members (63\%), mostly owners, with a medium-low socio-economic level (57\%). It is considered that housing is adequate in $93 \%$ of interviewees. The satisfaction with the complex comes from the fact that in most cases are the original owners. They accessed through the Public Employees Union and in most of the cases interviewed there is a significant period of permanence, with more than 30 years of residence in the neighborhood (Figure 2).

The parking area is only considered appropriate at $27 \%$ of cases. It is worth mentioning that only $33 \%$ have a car and that the original design of the complex provided for parking, as an extension on the perimeter streets. This criterion, inadequate due to the safety conditions of the property, results in spontaneous occupations of open spaces of common use. Even though the motorization index is very low, the parking areas invade collective spaces located on the perimeter streets to the blocks and nearby the dwellings in the ground floor.

$80 \%$ of the interviewees mentioned that they use public transport and are conform (have no complaints) with the travel times to the city centre. Although the complex has a peripheral location, over time this urban sector has reached an adequate level in terms of public services and infrastructure supply, modifying the initial situation of peripheral isolation, even though at the moment it is adjacent to other public housing complexes, some with levels of social conflict.

The evaluation of internal collective areas, as semi-closed city blocks is very satisfactory (91\%). It is explained given that these forms of resolution of the blocks allow the visual control of the central opened area from the dwellings units. It presents some degree of privacy in terms of use for the residents of each housing block. The level of maintenance of these open areas is regular, given that there is a low level of organization between the residents in the different consor- 
tia (blocks). In general, the maintenance of the courtyards is a voluntary work of the front residents to it. It is noteworthy that they are maintained as open spaces of free pedestrian accessibility (as projected in the 1980s). The large space as a central spine of the whole complex exceeds the possibility of maintenance by the inhabitants and is vandalized. Sociability is valued as good in $53 \%$ of cases, associated to the time living in the neighborhood and relates to the residential trajectory of the inhabitants in this group that are majority the original owners.

\subsection{CISPREN Complex}

CISPREN Complex has a medium density and a low RQI value.

Integrated by families in $87 \%$ of cases, 3 inhabitants (35\%) and 4 or more in $60 \%$ of cases; $13 \%$ are single-person households. Although this complex was awarded to members of the Trade Union Circle of Press workers, at the time of the study it presented $60 \%$ of owners and $40 \%$ of tenants. The socio-economic level of the inhabitants corresponds to medium-high sectors (with university studies in $53 \%$ of cases), Figure 2.

The assessment of the size of the dwelling was considered adequate at $80 \%$, and there is satisfaction with it according to the needs of the family group. This assessment is not consistent with the values obtained in the quantitative analysis. It is significant because this complex presents the lowest value of residential area per inhabitant and the departments are very small in size. However, when questioning about the choice to live in it, mention is made of the accessibility of the Colón corridor (a main avenue in the city) and the characteristics of the urban sector that has facilities at an urban scale, good accessibility and frequency on public transport lines presenting a good connectivity to the city centre.

A significant fact is that all the interviewees have a car however, $40 \%$ of the residents mention that they travel by public transport, and consider an important fact the urban location of the complex. The parking foresees only cover $40 \%$ of the cases interviewed. A perimeter fence was built which contributes to the greater security and privacy of the common central space. There is an increase in parking spaces in the outer perimeter of the complex built by residents. Those who do not have parking spaces inside the complex use the external docks or public streets.

Regarding the common space, the complex has a lounge (with grills) used by residents. It has central space very careful and well maintained used as a recreational park. There is a high assessment of internal sociability among the inhabitants (80\% answered that it is good) and also to the common areas/spaces that present a very positive assessment in terms of relationship.

The greater social homogeneity of the residents (middle or upper middle sectors), the resolution of the parking lots according to the demands raised, as well as the closure of the complex perimeter (that preserves the privacy and security conditions of the collective open areas), the clear definition of the urban form and a very careful and well maintained central area compensate the lower avail- 
ability of the internal area of the dwellings units.

\subsection{IPV Juniors Complex}

It has high density and a very low RQI value that is explained by the lower value of open space per inhabitant, compared to the other complexes and its greater building compactness (Figure 2).

Integrated by families in $85 \%$ of cases, 3 inhabitants (20\%) and $4(27 \%)$ and 5 or more in $33 \%$ of cases; $15 \%$ are single-person households. Although this complex was awarded by the Provincial Institute of Housing (IPV) for middle sectors (professionals) at the time of the study, it had $60 \%$ owners and $40 \%$ tenants. The socio-economic level of the population is medium-high (with university studies in $53 \%$ of cases).

The population conditions are similar to those of the CISPREN group.

There is a high degree of satisfaction with the dimensions of the housing units (93\% adequate) while a lower satisfaction is presented with the parking foreseen given that only $33 \%$ consider that is adequate. $53 \%$ have a car and keep it in the open spaces of the complex or in garages next to it that they rent for that purpose.

A different characteristic that was obtained in the interviews is that a low value of displacements in public transport was mentioned (33\%). The proximity to the city center and to the neighborhood centralities of the immediate surroundings evidences a greater amount of pedestrian displacements comparatively with other housing complexes. The common spaces, although smaller than in the other examples analyzed, are highly valued and used by residents, there is a high degree of maintenance and care of these areas (playgrounds, green areas, etc.) The level of sociability between neighbors is good (80\%) and the foreseen shops in the ground floors of the towers and blocks are valued as positive by the inhabitants.

This complex, where the RQI in quantitative terms expresses a low value, reaches a high level of satisfaction in terms of qualitative assessment. In this regard different conditions as to mention: the greatest social homogeneity, the organization of the resident consortium, the high level of maintenance and use of the open spaces and the conditions of urban location comparatively closer to the central area, to the mobility corridors and to the traditional neighborhood centralities in the city (such as the neighborhoods General Paz and Juniors).

\section{Final Comments}

A first reflection that emerges from the study is that the values of density do not have a direct correlation with the degree of satisfaction presented by the different models of spatial-physical organization of the collective housing complexes. That is, the hypothesis that a lower density has greater residential satisfaction and vice versa is not verified.

As we affirmed when developing the quantitative approach based on the si- 
mulation model, the analytical methodology we use offers the advantage of comparing dissimilar projects, and introducing temporary variations, given that the model is dynamic and allows us to study different responses when the plane/point of analysis varies. In the case of the study that we developed, the dynamic condition of the tool is given by the possibility of modifying the weights assigned to the different indicators, increasing or not their incidence in the final weighting of the residence quality index (RQI) expressed in a polynomial formula.

This potential of the tool makes possible to weigh tentatively the weight of one indicator over another, based on the values and aspirations that each social group of inhabitants expressed related to collective housing and obtain a dynamic response, introducing a more flexible vision when planning residential form. For example: Do the size of the housing units have the same impact on the formulation of a residential quality index as the open area of space per inhabitant offered by each architectural proposal? Or, to mention another example, which have a greater impact on residential satisfaction: the adequate foreseen of parking areas in the complex? Or the conditions that result from the urban location, accessibility to centralities and mobility in public transport?

The indicators and their weights were developed to obtain the values of the $\mathrm{RQI}$ and provide a comparative assessment, among the cases. That is, although they are relative, allow comparing the residential quality of a very different study universe in terms of formal-functional model and building density. The pending challenge, from a quantitative perspective, is the discussion of the standards (residential area per inhabitant, index of parking per dwelling, area of open space per inhabitant, among others) that should be applied in public housing design and planning. These should be agreed upon when planning residential interventions for collective housing, given that the demands and expectations are different depending on the social conditions of the population groups to which they are designed for. Some indicators as to mention: the socio-economic position; the composition and size of households, the life cycle of families, tenure and mobility conditions, should be integrated in the analysis of residential satisfaction.

The incorporation of qualitative assessment criteria in the analyzed housing complexes opens a series of subjective variables that make more complex the analytical model, linked to density and residential quality. Based on interviews with key informants and on-site observations, other data are obtained that incorporate the assessment of aspects not quantified initially, in the Residential Quality Index (RQI).

These qualitative aspects have a high incidence in the residential satisfaction and are explained in a particular way (own in each housing complex) given that they are aspects of a subjective nature linked to the social group that inhabits the complex.

The particular conditions of the inhabitants (valuations, expectations, preferences, habits, etc.) and the conditions of the urban structure where the complex 
is located (accessibility, mobility, security) became related in the model and integrated in the RQI.

The incorporation of qualitative indicators in the assessment of the levels of residential satisfaction of the housing complexes, has allowed to expand the discussion of the satisfiers that should be considered in the design of future residential proposals and to deepen the understanding of them, a complex situation given the differences that are presented in terms of quantitative indicators when comparing proposals addressed to different social of demand.

We agree with the authors mentioned in [8] that density is the most relevant variable, but not the only one to improve urban quality. The results of the comparison of the four complexes (low, medium and high density) allow us to recognize the importance of variables in the urban design of residential areas, as is the case of good accessibility from public transport corridors ; the accessibility to the services and social equipment in the immediate urban context, the configuration of the common open spaces of the complex with a scale according to the possibilities of maintenance of the consortium and with design conditions that offer security for the inhabitants.

Although the highest density is always associated with the location on corridors of public transport, the use made by the inhabitants of public transport depends on other conditions that are related to urban accessibility or socio-economic characteristics. In the study we observed that the largest displacements in public transport correspond to the groups that live in the complexes with the lowest density (case Hogar Propio Complex and SEP Complex where $87 \%$ and $80 \%$ respectively use public transport). The first case is explained by the conditions of location and proximity to the center (only 36 minutes by public transport) and in the second case, it is related to the lower socio-economic level of the residents given that only $33 \%$ of the interviewees have car.

It is significant that although all the residential complex are public management, in the densest groups (CISPREN and IPV Juniors) higher values in the levels of sociability among residents were obtained (80\% said it was good), even when the values of open space per inhabitants have the lower value compared to the other complexes ( 15.9 and $8 \mathrm{~m}^{2} /$ inhabitant, respectively). In these cases, the sociability or good level of coexistence among the inhabitants is linked to the design and maintenance of common spaces, rather than with the largest amount of open space. The higher socio-economic level of the residents allows the payment of maintenance expenses to keep in good conditions the common open spaces.

In those groups with the highest percentage of tenants (with respect to the original inhabitants), which implies greater residential mobility, a lower level of appropriation of the outdoor spaces of common use is expressed. In the SEP Complex (for example), the greatest satisfaction with the outer space of the proposal is linked to the sense of belonging and the time of residence in the complex, rather than to the conditions of maintenance and good treatment in those open areas. 
In general terms, it can be affirmed that satisfaction levels do not directly relate to the greater or lesser residential density of the architectural proposal, or to the size of the housing unit. In all the complexes, high values were obtained for valuing the size of the dwelling in relation to the composition of the households, $80 \%$ or more considered it adequate (although in the CISPREN complex, which is the one that presents smaller dwellings, the valuation is lower than in the other three cases). In this case the use and appropriation of the open area of the complex compensates for the reduced area the dwelling unit.

Other dimensions that affect residential satisfaction and are linked to the size of the housing typology are related to subjective aspects, such as the size and evolution of the family nucleus (which determines the availability of space inside the dwelling unit), or the lack of flexibility in the design of the units that do not allow incorporating transformations to adapt the space to the changing family needs.

In relation to parking spaces in the complexes, in all cases what was planned in the project is inadequate because the rate of parking per dwelling does not correspond to the demands of the family groups. In those complexes where high values were obtain (as in the case of Hogar Propio 93\% or 67\% CISPREN complex), the inhabitants expanded parking areas in the open spaces of the complex, and resolved the security conditions incorporating a limit in the perimeter. Currently it is verified that this is one of the most significant demands, to be attended that was not usually considered in the design of the collective public housing complexes.

Urban sustainability depends in a good way on density, and this to the residential quality with which we project collective housing. The compactness of the urban fabric is linked to the density, but also to the vitality, accessibility and quality of the projected open areas. The challenge then, is not to establish whether the densities should be high, medium or low, but to meet the design conditions of the complexes to allow formal and urban integration, respond to the diversity of households that integrate the demand, to generate social cohesion and offer residential quality.

\section{Conflicts of Interest}

The author declares no conflicts of interest regarding the publication of this paper.

\section{References}

[1] Marengo, M.C. (2017) Density and Housing: Comparative Evaluation towards a Residential Quality Index. The Case of Collective Dwellings in Córdoba-Argentina. Current Urban Studies, 5, 35-53. https://doi.org/10.4236/cus.2017.51003

[2] Shlomo, A., Jason, P., Daniel, L. and Blei, A. (2010) The Persistent Decline in Urban Densities: Global and Historical Evidence of "Sprawl".

https://www.lincolninst.edu/publications/working-papers/persistent-decline-urbandensities 
[3] Mozas, J. and Fernandez Per, A. (2006) Density Nueva Vivienda Colectiva. A+t Ediciones. España.

[4] Sabate, J. (2013) Mid-Rise, High-Density: To What Extent Does Density Matter? In: Proceedings Vertical Cities Asia International Design Competition \& Symposium, School of Design \& Environment, National University of Singapore, Singapur, 106-113.

[5] Marengo, M.C. (2013) Extensión urbana e intervenciones habitacionales. El caso de la ciudad de Córdoba (Argentina). https://ri.conicet.gov.ar/handle/11336/24668

[6] Glosario del Ministerio de Vivienda y Urbanismo. http://www.minvu.cl

[7] Universidad de Chile. Instituto de la Vivienda (2001) Estudio diagnóstico sistema de medición de satisfacción de beneficiarios de vivienda básica. Informe final. Facultad de Arquitectura y Urbanismo, Instituto de la Vivienda, Santiago, 7.

[8] Jacobs, J. (1973) Muerte y vida de las grandes ciudades. Ed. Península, Madrid.

[9] Aragones, J. and Amérigo, M. (1987) Satisfacción residencial: Un concepto de calidad de vida. Revista de Estudios Sociales y Sociología Aplicada. Documentación Social, Madrid, 67.

[10] Lefebvre, H. (1968) El derecho a la ciudad. Anthropos, París.

[11] Haramoto, E. (1994) Incentivo a la calidad de la vivienda social. http://www.revistainvi.uchile.cl/index.php/INVI/article/view/171/699

[12] Haramoto, E. (2002) Sistema medición satisfacción beneficiarios vivienda básica: Síntesis del informe de consultoría. Instituto de la Vivienda-División Técnica de Estudio y Fomento Habitacional. MINVU, FAU-UCH, Santiago. 